\title{
Development and Feasibility of an Electronic White Blood Cell Identification Trainer
}

\author{
DANIEL E. HAUN, ANGELA B. FOLEY, PATSY C. JARREAU
}

\begin{abstract}
A prototype computer-based training tool to improve WBC identification skills was developed. Students were assigned to complete five simulated WBC differentials but were allowed ample free time to use the tool at will to complete additional cases and to use the software in two alternative learning modes. The assignment was made at the end of the traditional WBC differential training activities in the first semester of hematology in the clinical laboratory science curriculum. The tool recorded usage data during the one month that students had access.
\end{abstract}

Student performance was compared to the consensus results from an expert panel of hematology instructors. Usage tracking data was extracted and reviewed. The performance data indicated that students varied in WBC identification skill on the assignment. The usage tracking data showed that students used the tool only slightly more than the assigned cases and did not use alternative learning modes.

Data from the expert panel indicated that the experts varied greatly in the number of discrepancies from the consensus opinion. Item analysis indicated the cell types that were most problematic.

The prototype experience prompted the creation of a revised subsequent version of the trainer that is now being evaluated in our CLS program. The new trainer is web-based offering personal computer and mobile device access.

ABBREVIATIONS: WBC-white blood cell; CLSClinical Laboratory Sciences; MLT-Medical Laboratory Technician; URL-universal resource locator (web address)

INDEX TERMS: Competency assessment; Student assessment; Psychomotor training; on-line training
Clin Lab Sci 2013;26(1):23

Daniel E. Haun MT(ASCP)H, LSU Health Sciences Center, Department of Clinical Laboratory Sciences, New Orleans, $L A$

Angela B. Foley MS, MLS(ASCP)SH, LSU Health Sciences Center, Department of Clinical Laboratory Sciences, New Orleans, LA

Patsy C. Jarreau MHS, MLS(ASCP), LSU Health Sciences Center, Department of Clinical Laboratory Sciences, New Orleans, LA

Address for Correspondence: Daniel E. Haun MT(ASCP)H, LSU Health Sciences Center, Department of Clinical Laboratory Sciences, 1900 Gravier St., New Orleans, LA 70112, 504.255.5848,dhaun@lsubsc.edu

\section{INTRODUCTION}

Diligently assessing practitioner's skills in WBC identification exposes practice gaps that go undetected with less rigorous competency assessment activities. ${ }^{1,2}$ Many labs use a selection of cell images, such as those from proficiency testing activities, to quiz employees as a method of competency assessment. Problems identified with this technique include the low number of cell examples used and possibility of "discussing" the correct answers by participants. ${ }^{2}$ Others have cited validity questions for these methods including content validity and predictive validity. ${ }^{1}$

The use of electronic tools for competency assessment has been explored and appears promising. ${ }^{2,3,4}$ Horiuchi et al used a commercially-produced competency software coupled with images from digital computeraided microscope system to assess competency and demonstrate improvement in poor performers. ${ }^{2}$ Burthem et al successfully piloted a digital morphology competency assessment method and delivered the package via the internet. Participant opinions of the 
method were generally favorable and participants indicated that they saw potential for applications of the technique as a teaching aid. ${ }^{4}$

Studies show that expertise leading to expert performance is gained by deliberate practice, which in a nutshell means practice in a manner of increasing challenge as skills are gained. ${ }^{5}$ Practicing at the same difficulty level over a long period of time will not lead to expert performance. Ericsson states that "living in cave does not make one a geologist", meaning that lowlevel practice and performance does not increase skill. He describes numerous studies showing performance gaps in medical skills and proposes that expertise in medicine could be improved by accessing a library of difficult challenges where one would perform diagnostic tasks and receive feedback from validated expert practitioners. $^{5}$

Tutoring is shown to improve student performance greatly over traditional classroom instruction. Bloom calls this the "2 sigma problem" because the demonstrated increase in performance is two standard deviations. It is a problem because tutoring is impractical for all students given the restraints of time and resources. He outlines the problem of finding teaching methods that would be equivalent to tutoring and suggests that computer-based training might be a solution. ${ }^{6}$ Studies have since confirmed this suggestion as a viable method of improving performance. ${ }^{7}$

In an ideal world, a student would learn WBC identification by a prolonged period of study with an expert but it is more likely that a student first learns identification with an instructor using projected images and talking through the identification process. This is typically followed by self-study on practice cases with some numerical data to compare one's performance, a limited number of assessments using study sets and adhoc consultation with the instructor on troublesome cells. In this way, the student becomes calibrated to the teacher and must meet a minimum level of proficiency, a variably defined grade of "C", before advancing.

In clinical training, students may encounter other faculty that may refine or recalibrate the student with such tools as double-headed microscope sessions and case studies that are assigned and discussed. Once in the workforce, the novice relies on peers, supervisors and doctoral staff for consultation and feedback. These are variable sources of unknown quality.

One limiting factor in these modes of learning is the variable skill level of the primary instructor, clinical instructors and peer consultants. The student or novice can never hope to progress beyond the skill level of the teacher. ${ }^{5}$ Validated expert feedback is a rare commodity in many settings, as in too many labs on too many shifts, technologists have limited face-to-face time with an expert instructor, pathologist or hematopathologist.

Given this background, an electronic tool that challenges users, allows for increasing difficulty as skills are gained and provides a standard for calibrating identification skills was envisioned and proposed. It would serve as a teaching and professional development tool to address the scarcity and variable nature of expert feedback in training and in the workforce. This study sought to explore the feasibility of developing such a tool.

\section{MATERIAL AND METHODS}

\section{Challenge Media}

Cell image sequences were captured using an analog charge-coupled device camera mounted to a light microscope. The camera's composite output was digitized using an analog to digital video converter (Grass Valley ADVC-55) and the digital output recorded on a personal computer using video recording software (QuickTime Player X. Apple Corporation, Cupertino, CA). A computer script was developed to collect video for exactly 7 seconds at 30 frames per second. Once recording started, the microscope was slowly focused up and down as one would when examining a cell. The resulting movie represented an image sequence (i.e. a "poor-man's z-stack") of 210 images that is easily delivered and used with ordinary web playback tools. During replay of the sequence, the user can simulate focus by manually manipulating the scrub bar of the video player.

Wright- stained peripheral blood smears were examined and white blood cells photographed in a systematic scan as if performing a 100 cell differential. The cells were not selected but taken in sequence to prevent selection bias and to provide a realistic representation of cells that the students would encounter in practice. 
In all, 2693 cell image sequences were collected from 25 cases that were grouped in five complexity levels composed of five cases each. Only the first 100 cells from each case were presented to the student and the others were considered spares for substitution in future applications. Complexity level one represented normal patients while level two represented cases with reactive lymphocytes. Level three represented cases exhibiting the granulocytic maturation series and levels four and five represented chronic and acute leukemic processes.

\section{Computer program description}

The basic function of the training software was to present the media, collect and evaluate the student's interpretation and give performance feedback when the case was finished. The prototype computer program was developed using FileMaker Pro 10 Advanced (FileMaker, Inc, Santa Clara, CA). The prototype featured interactive layouts for data and media display and used the FileMaker scripting language for programming interactivity. The programming details are complex and beyond the scope of this paper but the flowchart describes the simple functionality (Figure 1). The resulting software packages and media assets were then installed on seven computers in the student computer lab.

In the background, the prototype software tracked three key usage functions and allowed for password-protected extraction of the usage data. The trackers recorded the number of times that a student began a case, the number of times that they reviewed a missed cell and the number of times that they used the search function. Students were assigned five cases to complete, three from level two and two from level three and were instructed to submit printed results as proof of completion. The exercise was ungraded but points for completion of the assignment were counted toward the laboratory grade. The software was first demonstrated in the classroom by performing an example case as a group. The optional features, missed cell review and search of cells by cell type, were then demonstrated and discussed in a question and answer period. Students were encouraged to explore the software, to perform extra cases (other than those assigned) and to learn by searching and reviewing galleries of cell types. We wanted to know if the students would enjoy participation and use the tool to master cases beyond those levels that were assigned. The students were allowed one month to use the tool before the usage data was extracted.

Each student's results were analyzed to determine the number of discrepancies from the expert consensus (missed cells) for each case performed. The numbers were then totaled and the students ranked. Item analysis was performed to determine the cells that proved most problematic to the students.

Student evaluations of the tool were requested but not required. The form included questions on the quality and usefulness of the technique. Three open-ended questions allowed for praise, criticism and suggestions on how to improve the tool.

\section{Expert Opinion}

Prior to using the tool with students, experts evaluated the cells and submitted data. The expert data collection software was a modified version of the trainer software with additional form fields added to allow for comments and other specific metadata about the cell (e.g. toxic granulation, reactive lymphocyte, etc.). The

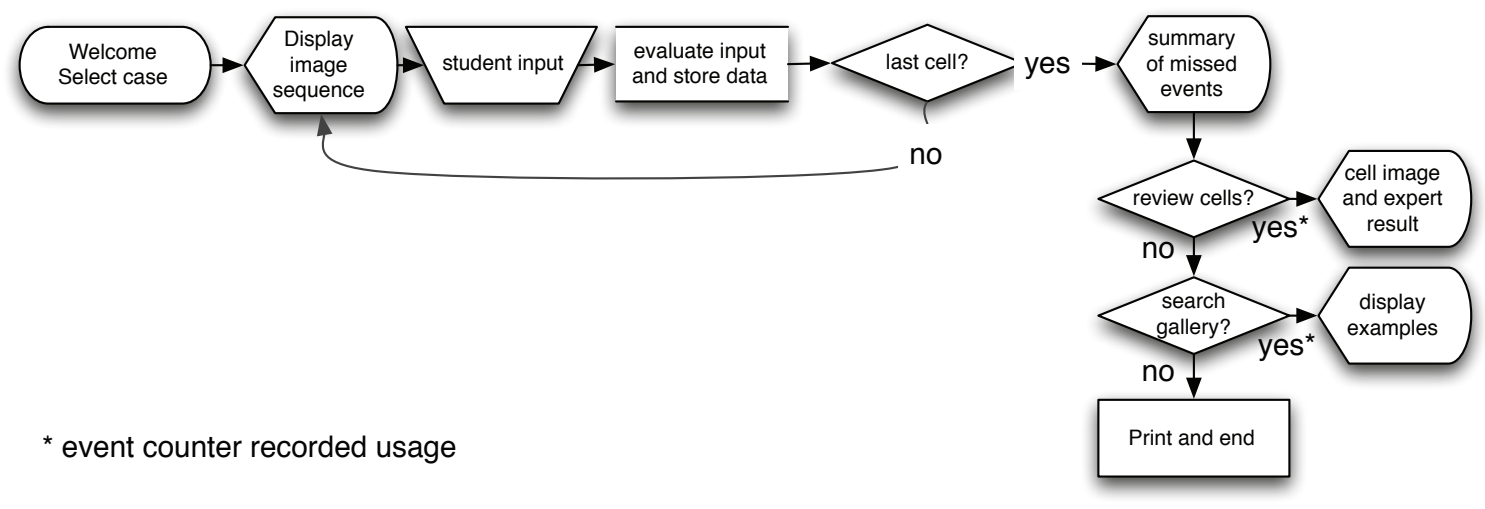

Figure 1. Flowchart of programming steps in the prototype version of the WBC identification trainer. 
experts were volunteers and represented hematology instructors from CLS and MLT programs. Twentynine teachers were recruited at the national Clinical Laboratory Educator's meeting but only seven participating experts represented programs in the Northeast (2),Midwest (3) and Southeast (2). The expert data was aggregated to determine a consensus answer that was used for evaluating the student's input. A consensus was determined to be the majority opinion when four or more experts agreed on the cell type. Full consensus was determined to be when all experts agreed. When a student chose to review a missed cell, all of the expert opinions and metadata was displayed along with the cell image sequence.

Each expert submission was evaluated to determine the number of discrepancies from the consensus opinion and these were used to rank the experts. Item analysis was also performed on this data to determine the cell types that proved to be problematic to the experts.

\section{RESULTS}

The ranking of students based on number of discrepancies showed that they varied in skill in identifying the cells presented by the tool. One student misidentified only 40 cells of the 500 represented in the exercise (8\%) but two students exceeded 90 misidentified cells (18\%). Figure 2 shows the distribution of student performance expressed by number of discrepancies from the expert consensus.

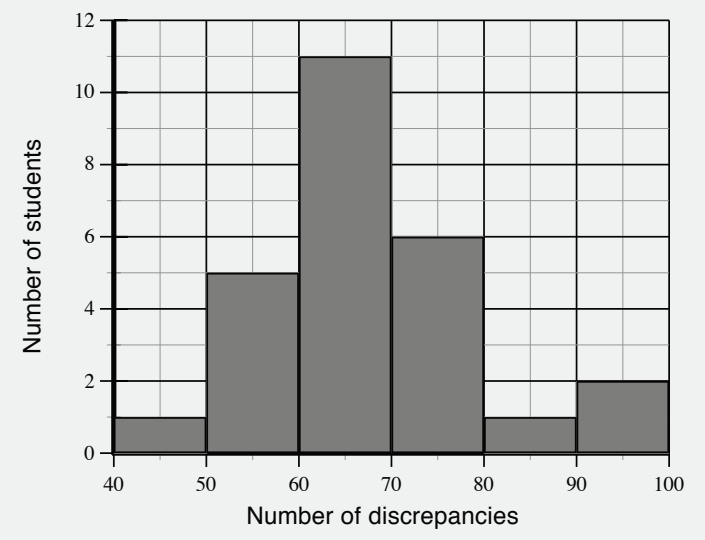

Figure 2. Bar graph showing the number of students (y -axis) by the number of discrepancies from the expert consensus ( $\mathrm{x}$ axis).
The most frequent discrepancy in this sample involved identification of large lymphocytes and monocytes but students also frequently mistook monocytes for bands and metamyelocytes. Degranulated basophils were also problematic as some students identified these as segmented neutrophils. A comprehensive profile of problematic cells seen in all 25 cases is not possible since only five cases from levels two and three were assigned in this study. The test sample did not contain any level 1 cases (normal) or any from levels 4 or 5 (containing cases of chronic and acute leukemia).

The usage data from the embedded counters showed 156 starts. All students completed all assigned cases for credit, which would require 130 starts. So the ratio of started cases to assigned cases was 1.2 to 1 . On average, each student started cases 6 times to complete the 5 assigned.

The total number of discrepancies for all students was 1738 and the tool tallied 718 requests for cell review, meaning that the students reviewed the missed cells $40 \%$ of the time. The tracker on the search function recorded a total of seven searches for all workstations in the one-month usage period.

The activity evaluation was completed by 16 of 26 students (62\%). The majority of responses were favorable and the open-ended responses were mostly general in nature but some revealed improvement opportunities. Specifically mentioned was the sharpness of the image, confusion when expert data showed disagreement and the desire to access the tool from home computers and mobile devices.

\section{Expert Results}

Sixty percent of cells had full consensus and 1\% (18 cells) did not have a consensus reached. Figure 3 illustrates the degree of expert consensus and percent of times that full consensus was not reached by number of outliers.

One expert differed from the consensus opinion only 22 times $(0.8 \%)$ but two participants differed at more than 10 times that rate $(8.9 \%$ and $9.7 \%)$. The other four participants differed from the consensus approximately $3 \%$ of the time. Figure 4 illustrates the number of times opinion differed from the consensus by expert. 


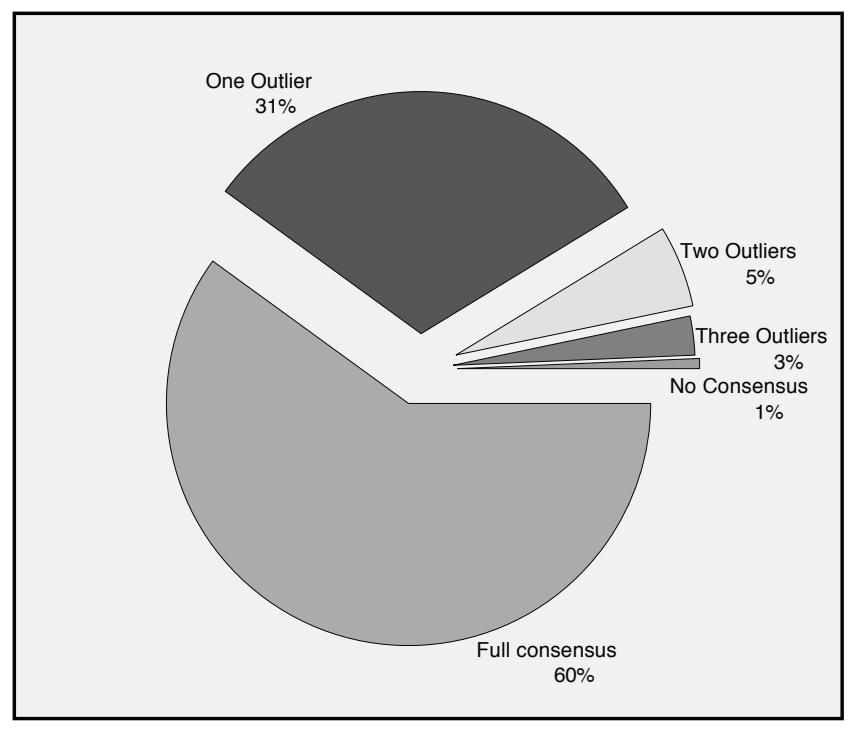

Figure 3. Pie chart demonstrating the percent of agreement between experts interpreting cells with the WBC identification trainer.

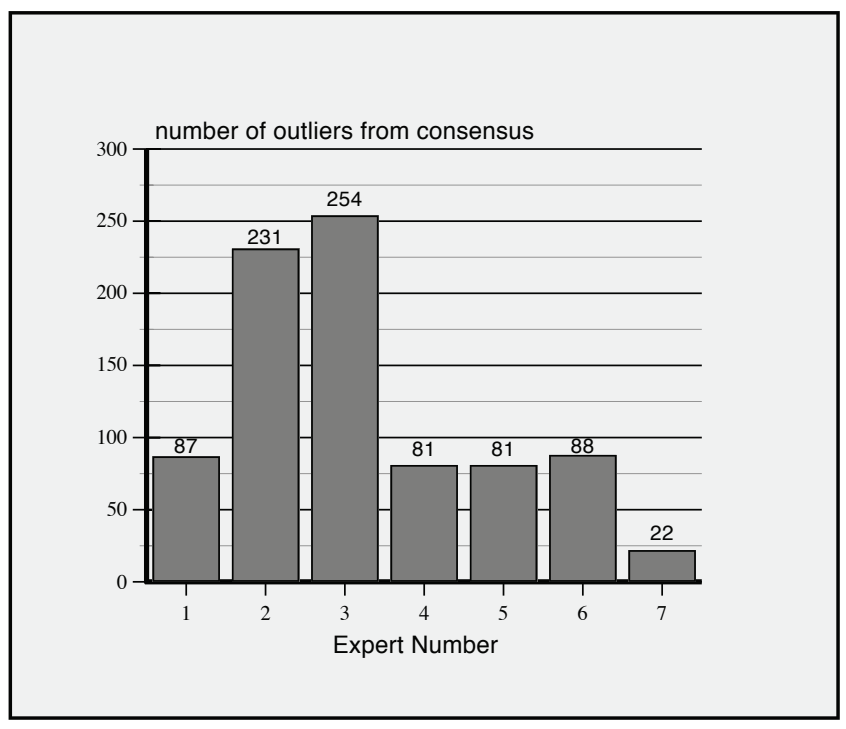

Figure 4. Bar graph indicating the number of discrepancies from the expert group consensus (y-axis) by individual expert (x-axis).

The identification of band versus segmented neutrophil was the most common case failing to get full consensus followed by blast cell identification, lymphocyte/ monocyte differentiation and the staging of neutrophil precursors. Figure 5 illustrates the categories of disagreement with percentages.

\section{DISCUSSION}

The performance data from both students and experts

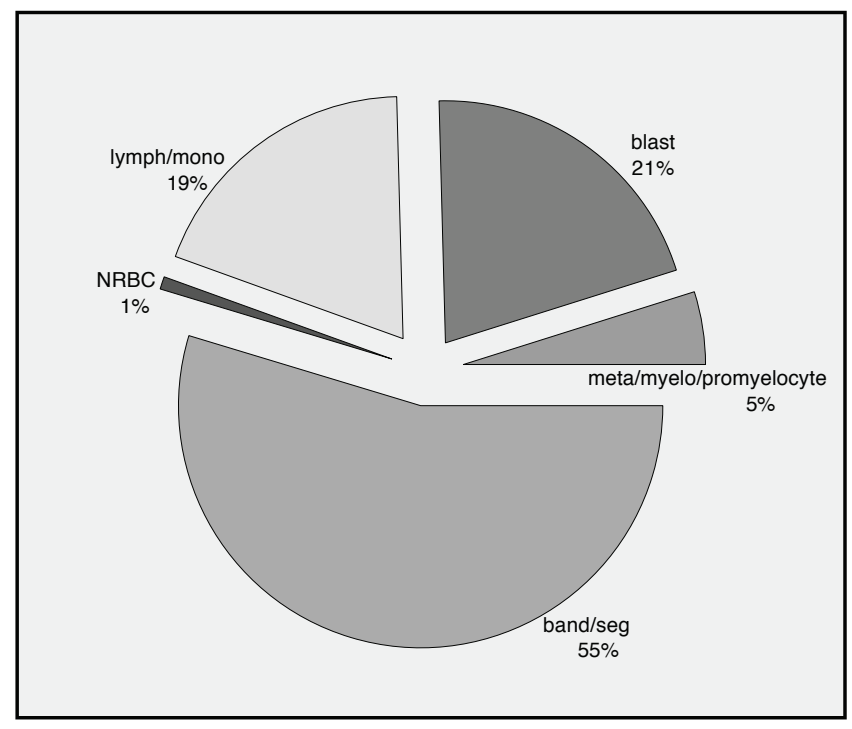

Figure 5. Pie chart demonstrating the categories of cells where the experts disagreed.

suggests that the tool can differentiate users' ability to identify WBCs. Some users show a low number of discrepancies while others exceed these by an order of magnitude. This finding was expected in the student group but somewhat surprising in the expert group.

The usage data indicates that students did not use the tool to master cases more difficult than those assigned. Practice and exploration was determined to be minimal at best because it is reasonable to believe that some extra starts were in fact "do-overs" or "start-overs". We conclude that assignments should be used to direct the student's progress.

Similarly, the relatively low rate of missed-cell review suggests that the review step should be automatic before allowing the student to proceed. This step came at the end of the case, was optional and was only used $40 \%$ of the time. It is our desire that the student take a second look at missed cells in light of the expert opinion as a method of calibration to the expert. Also the low number of searches performed, further supports the need to create assignments if searches are deemed desirable to learning. We conclude that the tool as designed is not fun for the students to use.

We are hesitant to over-analyze student evaluations from such a small number of participants but there were a number of negative ratings on image clarity and the written comments indicate a need to improve the image 
sharpness over the relative "fuzziness" (as noted by one student) seen in the image sequences. This "fuzziness" is presumed to be due to compression of the image in the movie making process. To address this issue, we now use a hybrid approach. A higher quality static image is initially displayed and the user clicks on the static image to call up the image sequence. We continue to offer the image sequence because some students reported favorably on the technique and we are hopeful that in the future, a technological advance will improve the clarity of the sequence. We want to continue to explore the use of this simple and efficient capture technique.

The student dissatisfaction with seeing the varying expert opinions is also addressed in the new version. Now, cells with more than one disagreeing expert are removed from the library and only the consensus opinion is shown to the student. The original design was to collect 100 cells without selection to eliminate bias and provide real world experience. But now the idea is to eliminate controversy, concentrate only on cells where identification is validated by near full consensus and consider adding back the more complex features as more experience is gained.

The expressed desire to make the tool available at home and on mobile devices is also addressed. The tool is now web based and all modern browsers based on web standards, including mobile browsers, will execute the program.

The new version also provides an assessment-only mode with all feedback steps deactivated but other functions left intact. So the teacher can assign either tutor mode or assessment mode by using a different URL.

\section{CONCLUSIONS AND RECOMMENDATIONS}

Weakness in using digital systems have been described including image quality, costs, ability to focus and selection bias. ${ }^{6}$ This project explored those weaknesses and weighed those against the reported advantages of image reproduction and distribution at distant sites at different times. The data suggests that the tool can differentiate users at both the novice and expert level, it can identify problematic cell types and it could have potential use in teaching and competency assessment.

The trade-offs considered in tool development are illustrated in this preliminary study and reveal many opportunities to improve the original design. As a desktop application, the tool required installation and limited the access to the computer lab. Too many cases were acquired in the initial design as we naively thought that the students would freely explore the other cases and master higher levels of complexity. We wasted a lot of time (and the time of our experts) to build such a large case library. The library may be useful for future projects. But since we only collected image sequences to build the first library and the new system uses a still image plus the image sequence, we had to start over and collect new media.

For the good parts, the study provided usage data and student opinion to instruct the development of subsequent versions. Also, the skills learned in developing the prototype gave the team confidence to learn the technologies necessary to web development.

With the web version, it is much easier to collect expert data and deploy a single case to students since all media and programs are installed on the web server instead of individual desktop computers. All programming parts are reusable and any updates reach all users automatically. The instructor now sends the assignment link via email and the results are emailed back to the instructor. The instructor can assign increasingly complex cases as the student progresses.

The new tool has proven to be robust thus far and serves as a practical adjunct to our current teaching methods. Item analysis identifies problematic cells and these can be brought to the classroom, projected and discussed. The tool differentiates user skill level and provides a basis from which improvement could be measured. The ability to detect poorer performance offers the potential for teachers (and lab managers) to customize remediation efforts for maximum effectiveness instead of doing one-size-fits-all training.

While the design is intended for hematology, the technique could be adapted to other disciplines such as microbiology and urinalysis where visual recognition skills are needed and where valid expert feedback may be obtained. In addition to use in student assessment, the tool might be used for research applications to answer questions about competency throughout the career of practitioners and to ensure the quality and consistency of faculty in teaching programs. 
This study and tool is very limited in addressing questions about microscopic skills. For instance, the simulator does not require microscope setup and since the images are displayed it does not address the searching and finding aspects of blood film examination. It only the addresses the interpret phase and only for the identification of the cell lineage and development stage of the white blood cells. The study does not show that the tool can improve performance but only seeks to establish a practical platform for measurement. Future studies will have to establish improvement to show that the tool has true value. Since all tool users know that they are being assessed, this tool only addresses the cognitive and psychomotor aspects of performance. It does not address the affective aspects necessary to real world performance and this might be better addressed with a study using retrospective review of real work.

In spite of this study's limitations, we believe that improving the skills in WBC identification is worthy of our efforts. To paraphrase Lord Kelvin, "if you can measure it, you can improve it" and while WBC identification is not the only element of peripheral blood film examination, it is a key part. It is reasonable to assume that health care organizations prefer experts to poor performers. Therefore, we conclude that it is reasonable to continue to seek tools that create experts and this type of tool is feasible to implement and worthy of future study.

\section{ACKNOWLEDGEMENTS}

The authors would like to acknowledge support from the Educational Enhancements Grants program of the LSUHSC-NO Academy for the Advancement of Educational Scholarship

\section{REFERENCES}

1. Haun DE, Leach AP, Fink DF, et al. A Better Way To Assess WBC Differential Counting Skills, Lab Med 2000;31(6):32933.

2. Horiuchi Y, Tabe Y, Idie, et al. The use of CellaVision competency software for external quality assessment and continuing professional development. J Clin Pathol 2011; 64:610-7

3. Brereton ML, De La Salle B., Burthem J, et al. Review of the UK NEQAS (H) digital morphology pilot scheme for continuing professional development accessed via the internet. Int. Jnl. Lab. Hem. 2008;30:365-71

4. Burthem J, Brereton M, Ardern J. The use of digital 'virtual slides' in the quality assessment of haematological morphology: results of a pilot exercise involving UK NEQAS(H) participants. Br J Haematol, 2005;130:293-6

5. Ericsson KA. Deliberate Practice and the Acquisition and Maintenance of Expert Performance in Medicine and Related Domains. Acad Med, 2004;79(10):S1-S12

6. Bloom BS. The 2 Sigma Problem: The Search for Methods of Group Instruction as Effective as One-to-One Tutoring. Ed Res, 1984;13(6):4-16

7. Corbett A. Cognitive Computer Tutors: Solving the TwoSigma Problem. Lecture Notes in Computer Science: Proceedings of the 8th International Conference on User Modeling, 2001;2109:137-47.

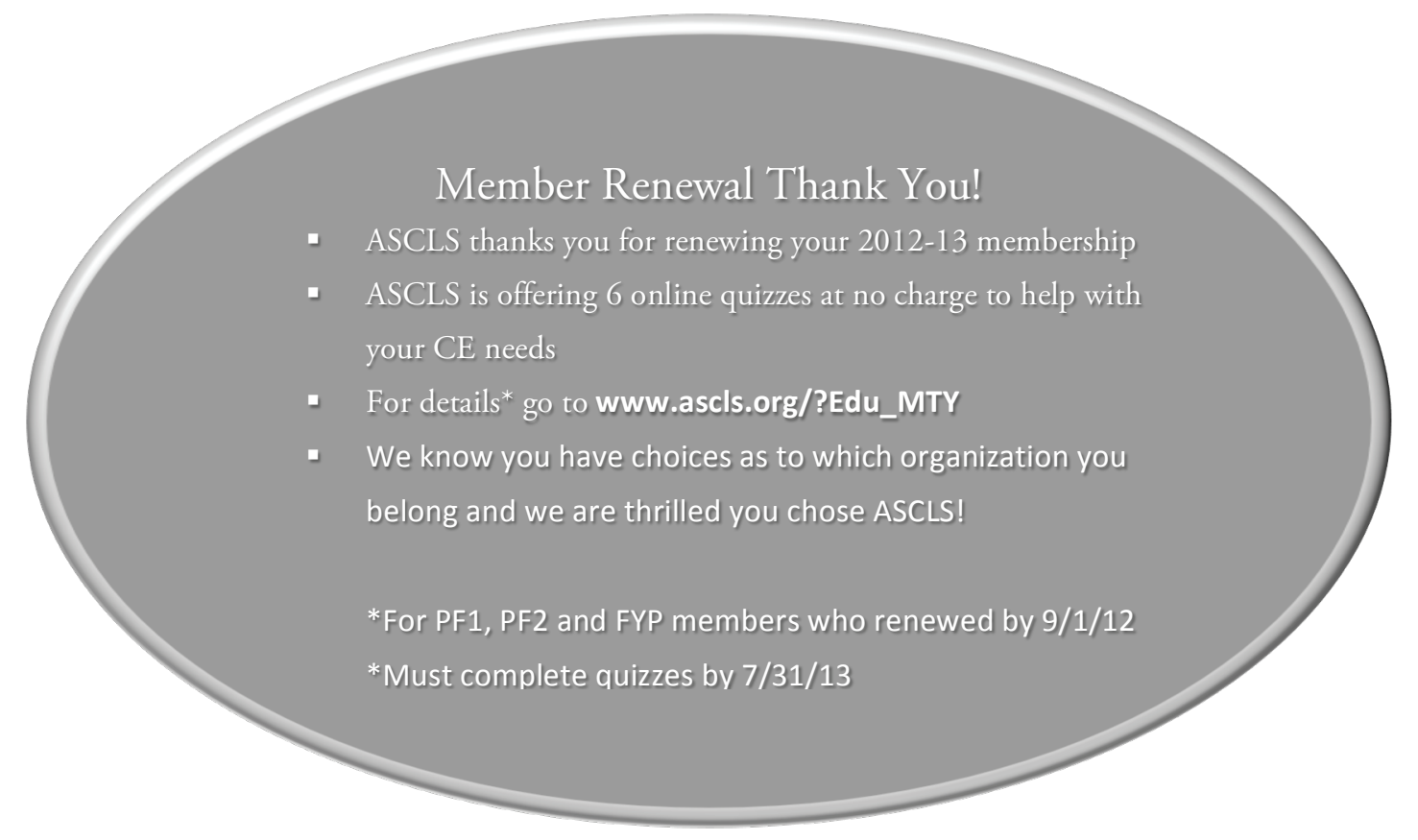

